\title{
DENSITY ORDER OF PARSEVAL WAVELET FRAMES FROM EXTENSION PRINCIPLES
}

\author{
A. SAN ANTOLÍN
}

\begin{abstract}
We characterize approximation order and density order of those Parseval wavelet frames obtained from Oblique Extension Principle. These notions are closely related to approximation order and density order by a quasi-projection operator. To give our characterizations, we shall explain the behavior on a neighborhood of the origin of the Fourier transform of a refinable function. In particular, we invoke the classical notion of approximate continuity. We write our results in the multivariate context of Parseval wavelet frames associated to $A$, an expansive linear map preserving the integer lattice.
\end{abstract}

\section{InTRODUCTION, NOTATION AND BASIC DEFINITIONS}

We are interested in approximation properties of wavelet frames. The aim of this paper is to give a characterization of approximation order and density order of those Parseval wavelet frames that can be constructed from Oblique Extension Principle. We also emphasis on approximation properties of a quasi-projection operator. Approximation order of shift invariant subspaces, quasi-projections operators and wavelet frames play a major role in the ability of wavelets and framelets to describe functions and in efficiency of some algorithm in applications as, for instance, signal processing and data compression. See e.g. the discussions in [13] and [18].

As far as the author knows, the notion of approximation order provided by wavelet frames has been firstly studied in [14], where the context is both classical on dimension one with the dyadic dilation and in the multivariate case with wavelet frames associated to isotropic dilation matrices. See also [15]. Approximation order provided by a Parseval wavelet frame from Oblique Extension Principle is closely related to approximation properties of a quasi-projection operator. Approximation properties of quasi-projection operators in $L^{2}$ have been obtained in [23], [24] and [3]. Here we give a characterization of approximation order provided by a Parseval wavelet frame constructed from Oblique Extension Principle and by a quasi-projection operator in the multivariate case where the dilation is given by an expansive linear map preserving the integer lattice. When the expansive linear map is not isotropic, we shall write the corresponding definitions and results in an adequate context using potential spaces associated to a pseudo-norm as an approximate subspace. Note that in the Lebesgue spaces $L^{p}$, related results have been proved in [28], [27], [8], [26] and [18], in weighted $L^{p}$ spaces, see [29]. Furthermore, approximation properties in Fourier algebras appeared in [16], in Besov spaces see [25] and in distribution spaces we refer to [31] and [30]. Often, approximation order

Key words and phrases. A-approximate continuity, approximation and density order, Extension Principles, Fourier transform, Parseval wavelet frame, quasi-projection operator.

2010 Mathematics Subject Classification: 42C15; 41A45; 41A63; 41A65. 
of shift-invariant spaces is related to polynomial reproduction properties, this is discussed, for instance, in [21].

Perhaps, our main advance in this paper is our characterization of density order provided by a Parseval wavelet frame constructed from Oblique Extension Principle, and density order provided by a quasi-projection operator. These notions have been motivated by the corresponding definition for a shift-invariant subspace given in [4]. For more results on best approximation of a shift invariant subspace in $L^{2}$, see e.g. $[3],[5],[36]$ and [11]. Let us mention that approximation properties of nonstationary wavelet frames are studied in [22]. Although the main results we prove here are new in the classical setting, we will work on the multivariate context.

Let us first write some notation. The sets of strictly positive integers, integers, real and complex numbers will be denoted by $\mathbb{N}, \mathbb{Z}, \mathbb{R}$ and $\mathbb{C}$ respectively. $\mathbb{T}^{d}=\mathbb{R}^{d} / \mathbb{Z}^{d}, d \geq 1$, and with some abuse of the notation we consider also that $\mathbb{T}^{d}$ is the unit cube $[0,1)^{d}$.

Given $r>0$, we will denote $B_{r}(\mathbf{y})=\left\{\mathbf{x} \in \mathbb{R}^{d}:|\mathbf{x}-\mathbf{y}|<r\right\}$, and will write $B_{r}$ if $\mathbf{y}$ is the origin. For a set $E \subset \mathbb{R}^{d}$ we will denote $E^{c}=\mathbb{R}^{d} \backslash E$ and if we write $\chi_{E}$, we mean the characteristic function of the set $E$, i.e. $\chi_{E}(\mathbf{t})=1$ if $\mathbf{t} \in E$ and $\chi_{E}(\mathbf{t})=0$ if $\mathbf{t} \in E^{c}$. Moreover, for a point $\mathbf{y} \in \mathbb{R}^{d}, \mathbf{y}+E=\{\mathbf{y}+\mathbf{x}:$ for $\mathbf{x} \in E\}$. We will consider that the sets $E \subset \mathbb{R}^{d}$ are (Lebesgue) measurable and the Lebesgue measure of $E \subset \mathbb{R}^{d}$ will be denoted as $|E|$. The spaces $L^{p}\left(\mathbb{R}^{d}\right), 1 \leq p \leq \infty$ will be the corresponding standard Lebesgue spaces of complex-valued measurable functions. Moreover, if we write $f \in L^{p}\left(\mathbb{T}^{d}\right)$ we will also mean that $f$ is defined on the whole space $\mathbb{R}^{d}$ as an $\mathbb{Z}^{d}$-periodic function.

Let us consider $f, g \in L^{2}\left(\mathbb{R}^{d}\right)$. The bracket product of functions $f$ and $g$ is defined by

$$
[f, g](\mathbf{x})=\sum_{\mathbf{k} \in \mathbb{Z}^{d}} f(\mathbf{x}+\mathbf{k}) \overline{g(\mathbf{x}+\mathbf{k})}
$$

where it makes sense. We will denote

$$
\mathcal{N}_{f}=\left\{\mathbf{x} \in \mathbb{R}^{d}:[f, f](\mathbf{x})=0\right\} .
$$

The sets are defined modulo a null measurable set and we understand some equations as almost everywhere on $\mathbb{R}^{d}$ or $\mathbb{T}^{d}$. Moreover we consider $\frac{0}{0}=0$ or $0 \frac{1}{0}=0$ in the expressions where such indeterminacy appear.

A key tool in the study of wavelet frames is the Fourier transform. Here, our convention is that if $f \in L^{1}\left(\mathbb{R}^{d}\right)$,

$$
\widehat{f}(\mathbf{x}):=\int_{\mathbb{R}^{d}} f(\mathbf{t}) e^{-2 \pi i \mathbf{t} \cdot \mathbf{x}} d \mathbf{t}
$$

where $\mathbf{t} \cdot \mathbf{x}$ denotes the usual inner product of vectors $\mathbf{t}$ and $\mathbf{x}$ in $\mathbb{R}^{d}$. The definition of Fourier transform is extended as usual to functions in $L^{2}\left(\mathbb{R}^{d}\right)$.

With some abuse in the notation, given a linear invertible map $A: \mathbb{R}^{d} \longrightarrow \mathbb{R}^{d}$ we will also denote by $A$ the corresponding matrix associated with the canonical basis. Moreover, we write $d_{A}=|\operatorname{det} A|$ and the adjoint of the linear map $A$ will be denoted by $A^{*}$. Given a measurable set $E \subset \mathbb{R}^{d}$, then $A(E)=\{A(\mathbf{x}): \mathbf{x} \in E\}$ and the volume of $E$ changes under $A$ according to $|A(E)|=d_{A}|E|$. Recall that a linear map $A$ is said to be expansive if the modulus of all (complex) eigenvalues of $A$ is greater than 1 . If $A$ is a corresponding matrix of an expansive linear map, 
then obviously $d_{A}>1$. When the modulus of all the eigenvalues of $A$ is the same, then $A$ is usually called an isotropic linear map.

For a given linear invertible map $A: \mathbb{R}^{d} \rightarrow \mathbb{R}^{d}$ consider the unitary operator $D_{A}: L^{2}\left(\mathbb{R}^{d}\right) \rightarrow L^{2}\left(\mathbb{R}^{d}\right)$ defined by

$$
D_{A} f(\cdot)=d_{A}^{1 / 2} f(A \cdot), \quad \text { for any } \quad f \in L^{2}\left(\mathbb{R}^{d}\right) .
$$

It can be easily verified that

$$
D_{A}^{-1}=D_{A^{-1}}
$$

For any $\mathbf{u} \in \mathbb{R}^{d}$, let $\tau_{\mathbf{u}}: L^{2}\left(\mathbb{R}^{d}\right) \rightarrow L^{2}\left(\mathbb{R}^{d}\right)$ be the operator of translation by the vector $\mathbf{u}$ defined by

$$
\tau_{\mathbf{u}} f(\cdot)=f(\cdot-\mathbf{u}), \quad \text { for any } \quad f \in L^{2}\left(\mathbb{R}^{d}\right) .
$$

In addition, given $j \in \mathbb{Z}$ and a set $S$ of $L^{2}\left(\mathbb{R}^{d}\right)$,

$$
D_{A}^{j}(S)=\left\{d_{A}^{j / 2} f\left(A^{j} \cdot\right): f \in S\right\} \quad \text { and } \quad \tau(S)=\left\{f(\cdot-\mathbf{k}): f \in S, \mathbf{k} \in \mathbb{R}^{d}\right\} .
$$

If $A: \mathbb{R}^{d} \rightarrow \mathbb{R}^{d}$ is an expansive linear map such that $A\left(\mathbb{Z}^{d}\right) \subset \mathbb{Z}^{d}$, then the quotient groups $\mathbb{Z}^{d} / A\left(\mathbb{Z}^{d}\right)$ and $\left(A^{*}\right)^{-1}\left(\mathbb{Z}^{d}\right) / \mathbb{Z}^{d}$ are well defined. Recall that there are exactly $d_{A}$ cosets of $\mathbb{Z}^{d} / A\left(\mathbb{Z}^{d}\right)$ (see $[17]$ ). Thus there are exactly $d_{A}$ cosets of $\left(A^{*}\right)^{-1}\left(\mathbb{Z}^{d}\right) / \mathbb{Z}^{d}$.

Let $A: \mathbb{R}^{d} \rightarrow \mathbb{R}^{d}$ be an expansive linear map such that $A\left(\mathbb{Z}^{d}\right) \subset \mathbb{Z}^{d}$. Let $\Psi=\left\{\psi^{(1)}, \ldots, \psi^{(N)}\right\} \subset L^{2}\left(\mathbb{R}^{d}\right)$ be a set of functions, we call

$$
X_{\Psi}:=\left\{D_{A}^{j} \tau_{\mathbf{k}} \psi^{(\ell)}: j \in \mathbb{Z}, \mathbf{k} \in \mathbb{Z}^{d}, 1 \leq \ell \leq N\right\}
$$

the affine system generated by $\Psi$. The set of functions $\Psi$ is called a Parseval wavelet frame or a Parseval framelet associated to $A$ for $L^{2}\left(\mathbb{R}^{d}\right)$ when

$$
f(\mathbf{x})=\sum_{\ell=1}^{N} \sum_{j \in \mathbb{Z}} \sum_{\mathbf{k} \in \mathbb{Z}^{d}}\left\langle f, D_{A}^{j} \tau_{\mathbf{k}} \psi^{(\ell)}\right\rangle D_{A}^{j} \tau_{\mathbf{k}} \psi^{(\ell)}(\mathbf{x})
$$

where the convergence is in $L^{2}\left(\mathbb{R}^{d}\right)$. Here $\langle\cdot, \cdot\rangle$ denotes the inner product on $L^{2}\left(\mathbb{R}^{d}\right)$. The functions $\psi^{(\ell)}, \ell=1, \ldots, N$ are called the generators of the Parseval wavelet frame. When the context is clear we do not write "associated to $A$ ".

Wavelet frames have been widely studied in the literature, see e.g. the book [9]. In the multivariate case, we refer the extensive study done in [30] and [18].

The following definitions have been introduced in [10].

Definition 1. Let $A: \mathbb{R}^{d} \rightarrow \mathbb{R}^{d}$ be an expansive linear map. It is said that $\mathbf{x} \in \mathbb{R}^{d}$ is a point of $A$-density for a measurable set $E \subset \mathbb{R}^{d},|E|>0$, if for any $r>0$

$$
\lim _{j \rightarrow \infty} \frac{\left|E \cap\left(A^{-j} B_{r}+\mathbf{x}\right)\right|}{\left|A^{-j} B_{r}\right|}=1 .
$$

Definition 2. Let $A: \mathbb{R}^{d} \rightarrow \mathbb{R}^{d}$ be an expansive linear map. Let $f: \mathbb{R}^{d} \longrightarrow \mathbb{C}$ be a measurable function. It is said that $\mathbf{x} \in \mathbb{R}^{d}$ is a point of $A$-approximate continuity of the function $f$ if there exists $E \subset \mathbb{R}^{d},|E|>0$, such that $\mathbf{x}$ is a point of $A$-density for the set $E$ and

$$
\lim _{\substack{\mathbf{y} \rightarrow \mathbf{x} \\ \mathbf{y} \in E}} f(\mathbf{y})=f(\mathbf{x})
$$


Remark 1. If $A$ is isotropic, the notion of $A$-approximate continuity coincide with the classical notion of approximate continuity, see e.g. [33] and [6].

This paper is organized as follows: In Section 2 we introduce the notion of approximation order and density order for a Parseval wavelet frame associated to a general dilation $A$ as well as for quasi-projections operators. In addition, we recall a version of Oblique Extension Principle. The main results of this paper can be found in Section 3 while their proofs are postponed to Section 4.

\section{Density order of a PARseval WaVelet Frame}

In this section, we introduce the definition of $A$-approximation order and $A$ density order provided by quasi-projection operators and by a Parseval wavelet frame associated to a dilation $A$. These generalize the well-known notions given in [14]. Afterwards, we recall those Parseval wavelet frame constructed from Oblique Extension Principle.

2.1. Approximation order and density order. We start writing an adequate context. For this purpose, we need the following definition introduced in [12]. A measurable mapping $\rho: \mathbb{R}^{d} \rightarrow[0, \infty)$ is a pseudo-norm associated to the expansive linear map $A: \mathbb{R}^{d} \rightarrow \mathbb{R}^{d}$ if

(i) $\rho$ is $C^{\infty}$ on $\mathbb{R}^{d} \backslash\{\mathbf{0}\}$;

(ii) $\rho(\mathbf{x})=0 \Longleftrightarrow \mathbf{x}=\mathbf{0}$;

(iii) $\rho(-\mathbf{x})=\rho(\mathbf{x})$;

(iv) $\rho(A \mathbf{x})=d_{A}^{1 / d} \rho(\mathbf{x})$.

Moreover, the authors of [12] build one of them and observe that pseudo-norms for an $A$ are unique up to equivalence.

For $A: \mathbb{R}^{d} \rightarrow \mathbb{R}^{d}$ an expansive linear map, $\rho$ a pseudo-norm associated to $A^{*}$ and $\alpha \geq 0$, we will consider the Hilbert space

$$
W_{A}^{\alpha, 2}\left(\mathbb{R}^{d}\right)=\left\{f \in L^{2}\left(\mathbb{R}^{d}\right):\left\|(1+\rho)^{\alpha} \widehat{f}\right\|_{L^{2}\left(\mathbb{R}^{d}\right)}<\infty\right\}
$$

with norm defined by

$$
\|f\|_{W_{A}^{\alpha, 2}\left(\mathbb{R}^{d}\right)}=\left\|(1+\rho)^{\alpha} \widehat{f}\right\|_{L^{2}\left(\mathbb{R}^{d}\right)} .
$$

Note that for any two pseudo-norms associated to $A^{*}$ the corresponding spaces are equivalents.

From here to the end of this paper, we fix $A: \mathbb{R}^{d} \rightarrow \mathbb{R}^{d}$, an expansive linear map such that $A \mathbb{Z}^{d} \subset \mathbb{Z}^{d}, \rho$ a pseudo-norm associated to $A^{*}$ and $\alpha \geq 0$. Addition, we fix $\Omega_{A}=\left\{\mathbf{p}_{k}\right\}_{k=0}^{d_{A}-1}$ a full collection of representatives of the cosets of $\left(A^{*}\right)^{-1}\left(\mathbb{Z}^{d}\right) / \mathbb{Z}^{d}$.

We need the following operators. For a given $\phi \in L^{2}\left(\mathbb{R}^{d}\right)$ such that $[\widehat{\phi}, \widehat{\phi}]$ is essentially bounded, the quasi-projection operator at level $n \in \mathbb{N}$ is defined by

$$
P_{\phi, A, n}(f)=\sum_{\mathbf{k} \in \mathbb{Z}^{d}}\left\langle f, D_{A}^{n} \tau_{\mathbf{k}} \phi\right\rangle D_{A}^{n} \tau_{\mathbf{k}} \phi, \quad f \in L^{2}\left(\mathbb{R}^{d}\right) .
$$

Since $[\widehat{\phi}, \widehat{\phi}] \in L^{\infty}\left(\mathbb{T}^{d}\right)$, it is not hard to see that $P_{\phi, A, n}$ defines a bounded linear operator from $L^{2}\left(\mathbb{R}^{d}\right)$ to $L^{2}\left(\mathbb{R}^{d}\right)$ with norm $\left\|P_{\phi, A, n}\right\| \leq\|[\widehat{\phi}, \widehat{\phi}]\|_{L^{\infty}\left(\mathbb{T}^{d}\right)}$. For explicit computations, we refer to [30, Lemma 2.2.3]. 
If we consider a set of functions $\Psi=\left\{\psi^{(\ell)}: \ell=1, \ldots, N\right\} \subset L^{2}\left(\mathbb{R}^{d}\right)$ where $X_{\Psi}$ is a Parseval wavelet frame of $L^{2}\left(\mathbb{R}^{d}\right)$, the truncated representation at level $n \in \mathbb{N}$, is defined by

$$
Q_{\Psi, A, n}(f)=\sum_{\ell=1}^{N} \sum_{\mathbf{k} \in \mathbb{Z}^{d}} \sum_{j=-\infty}^{n-1}\left\langle f, D_{A}^{j} \tau_{\mathbf{k}} \psi^{(\ell)}\right\rangle D_{A}^{j} \tau_{\mathbf{k}} \psi^{(\ell)}, \quad f \in L^{2}\left(\mathbb{R}^{d}\right),
$$

where the convergence is in $L^{2}\left(\mathbb{R}^{d}\right)$ and $\langle\cdot, \cdot\rangle$ denotes the inner product in $L^{2}\left(\mathbb{R}^{d}\right)$.

We introduce the notions of linear approximation order and density order with a dilation matrix of the operators $P_{\phi, A, n}$. In dimension one and with the dyadic dilation, we refer to [24].

Definition 3. Let $\phi \in L^{2}\left(\mathbb{R}^{d}\right)$ such that $[\widehat{\phi}, \widehat{\phi}] \in L^{\infty}\left(\mathbb{T}^{d}\right)$. The operators $P_{\phi, A, n}$, $n \in \mathbb{N}$, provide A-approximation order $\alpha$, if there exists a constant $C>0$ such that $\left\|f-P_{\phi, A, n}(f)\right\|_{L^{2}\left(\mathbb{R}^{d}\right)} \leq C d_{A}^{-n \alpha / d}\|f\|_{W_{A}^{\alpha, 2}\left(\mathbb{R}^{d}\right)}, \quad \forall f \in W_{A}^{\alpha, 2}\left(\mathbb{R}^{d}\right), \quad \forall n \in \mathbb{N}$.

Definition 4. Let $\phi \in L^{2}\left(\mathbb{R}^{d}\right)$ such that $[\widehat{\phi}, \widehat{\phi}] \in L^{\infty}\left(\mathbb{T}^{d}\right)$. The operators $P_{\phi, A, n}$, $n \in \mathbb{N}$, provide $A$-density order $\alpha$, if

$$
\lim _{n \rightarrow \infty} d_{A}^{n \alpha / d}\left\|f-P_{\phi, A, n}(f)\right\|_{L^{2}\left(\mathbb{R}^{d}\right)}=0 \quad \forall f \in W_{A}^{\alpha, 2}\left(\mathbb{R}^{d}\right) .
$$

Now, we introduce the notions of approximation order and density order of a Parseval wavelet frame associated to a dilation matrix. See [14] for isotropic dilation matrices.

Definition 5. A Parseval wavelet frame $\Psi=\left\{\psi^{(\ell)}: \ell=1, \ldots, N\right\} \subset L^{2}\left(\mathbb{R}^{d}\right)$, provides $A$-approximation order $\alpha$, if there exists a constant $C>0$ such that

$\left\|f-Q_{\Psi, A, n}(f)\right\|_{L^{2}\left(\mathbb{R}^{d}\right)} \leq C d_{A}^{-n \alpha / d}\|f\|_{W_{A}^{\alpha, 2}\left(\mathbb{R}^{d}\right)}, \quad \forall f \in W_{A}^{\alpha, 2}\left(\mathbb{R}^{d}\right), \quad \forall n \in \mathbb{N}$.

Definition 6. A Parseval wavelet frame $\Psi=\left\{\psi^{(\ell)}: \ell=1, \ldots, N\right\} \subset L^{2}\left(\mathbb{R}^{d}\right)$, provides $A$-density order $\alpha$, if

$$
\lim _{n \rightarrow \infty} d_{A}^{n \alpha / d}\left\|f-Q_{\Psi, A, n}(f)\right\|_{L^{2}\left(\mathbb{R}^{d}\right)}=0 \quad \forall f \in W_{A}^{\alpha, 2}\left(\mathbb{R}^{d}\right) .
$$

Remark 2. Note that when $P_{\phi, A, n}$ provides A-density order $\alpha$, this implies that $P_{\phi, A, n}$ provides A-approximation order $\alpha$. This is a consequence of the BanachSteinhaus Theorem. By the same argument, that a Parseval wavelet frame $\Psi$ provides $A$-density order $\alpha$ implies that $\Psi$ provides $A$-approximation order $\alpha$.

2.2. Parseval wavelet frames from OEP. Unitary Extension Principle (UEP) and Oblique Extension Principle (OEP), a more flexible method based on the UEP, are very useful tools for constructing wavelet frames. References on different versions of these principles are e.g. [34], [2], [7], [14], [20, 19, 18], [1], [32] and [35].

To prove our main result, we need the following version of Oblique Extension Principle, see [35].

Theorem A. Let $\phi \in L^{2}\left(\mathbb{R}^{d}\right)$ such that

$$
\widehat{\phi}\left(A^{*} \mathbf{t}\right)=H_{0}(\mathbf{t}) \widehat{\phi}(\mathbf{t}), \quad \text { a.e., }
$$

where $H_{0} \in L^{\infty}\left(\mathbb{T}^{d}\right)$. Moreover, let $S$ be a non-negative $\mathbb{Z}^{d}$-periodic measurable function such that $S[\widehat{\phi}, \widehat{\phi}] \in L^{\infty}\left(\mathbb{T}^{d}\right)$, and also, the origin is a point of $A^{*}$ approximate continuity for $S|\widehat{\phi}|^{2}$, assumed $S(\mathbf{0})|\widehat{\phi}(\mathbf{0})|^{2}=1$. Let $H_{1}, \ldots, H_{N} \in$ 
$L^{\infty}\left(\mathbb{T}^{d}\right)$ such that

$$
S\left(A^{*} \mathbf{t}\right)\left|H_{0}(\mathbf{t})\right|^{2}+\sum_{\ell=1}^{N}\left|H_{\ell}(\mathbf{t})\right|^{2}=S(\mathbf{t}) \quad \text { a.e. } \quad \mathbf{t} \in \mathbb{R}^{d} \backslash \mathcal{N}_{\widehat{\phi}}
$$

and the equality

$$
S\left(A^{*} \mathbf{t}\right) H_{0}(\mathbf{t}) \overline{H_{0}\left(\mathbf{t}+\mathbf{p}_{k}\right)}+\sum_{\ell=1}^{N} H_{\ell}(\mathbf{t}) \overline{H_{\ell}\left(\mathbf{t}+\mathbf{p}_{k}\right)}=0
$$

holds for a.e. $\mathbf{t} \in \mathbb{R}^{d} \backslash \mathcal{N}_{\widehat{\phi}}$ and for any $\mathbf{p}_{k}, k=1, \ldots, d_{A}-1$, such that $\mathbf{t}+\mathbf{p}_{k} \in$ $\mathbb{R}^{d} \backslash \mathcal{N}_{\widehat{\phi}}$.

$$
\begin{aligned}
& \text { Define } \psi_{1}, \ldots, \psi_{N} \in L^{2}\left(\mathbb{R}^{d}\right) \text { by } \\
& \qquad \widehat{\psi_{\ell}}\left(A^{*} \mathbf{t}\right)=H_{\ell}(\mathbf{t}) \widehat{\phi}(\mathbf{t}) \quad \text { a.e., } \quad \ell=1, \ldots, N .
\end{aligned}
$$

Then the set of functions $\Psi=\left\{\psi^{(\ell)}: \ell=1, \ldots, N\right\}$ is a Parseval wavelet frame of $L^{2}\left(\mathbb{R}^{d}\right)$.

\section{Main Results}

In this section we collect the main results of this paper. The proofs are postponed to the next section.

Our results on approximation order and density order depend on the behavior near the origin of two functions as the following. For a $\varphi \in L^{2}\left(\mathbb{R}^{d}\right)$ such that $[\widehat{\varphi}, \widehat{\varphi}] \in L^{\infty}\left(\mathbb{R}^{d}\right)$, we define

$$
\Gamma_{\varphi, A, \alpha}(\mathbf{t}):=\left\{\begin{array}{ccc}
\left.\rho(\mathbf{t})^{-\alpha}|1-| \widehat{\varphi}(\mathbf{t})\right|^{2} \mid & \text { if } \quad \begin{array}{c}
\mathbf{t} \in \mathbb{R}^{d} \backslash\{\mathbf{0}\} \\
0
\end{array} \\
\mathbf{t}=\mathbf{0}
\end{array}\right.
$$

and

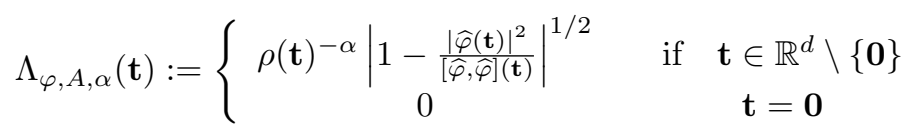

Observe that always $\Gamma_{\varphi, A, \alpha}(\mathbf{0})=\Lambda_{\varphi, A, \alpha}(\mathbf{0})=0$.

The following is a characterization of approximation order provided by quasiprojection operators.

Theorem 1. Let $\phi \in L^{2}\left(\mathbb{R}^{d}\right)$ such that $[\widehat{\phi}, \widehat{\phi}]$ is essentially bounded. The operators $P_{\phi, A, n}, n \in \mathbb{N}$, provide A-approximation order $\alpha$ if and only if both

$$
\Lambda_{\phi, A, \alpha} \quad \text { and } \Gamma_{\phi, A, \alpha} \text { are in } L^{\infty}\left(\mathbb{R}^{d}\right) .
$$

As a consequence, we have the following characterization of approximation order provided by a Parseval wavelet frame constructed from Oblique Extension Principle.

Corollary 1. With the same hypotheses as in Theorem A. The Parseval wavelet frame $\Psi=\left\{\psi^{(\ell)}: 1 \leq \ell \leq N\right\}$ provides A-approximation order $\alpha$ if and only if both

$$
\Lambda_{\sqrt{S} \phi, A, \alpha} \text { and } \quad \Gamma_{\sqrt{S} \phi, A, \alpha} \text { are in } L^{\infty}\left(\mathbb{R}^{d}\right) .
$$

Perhaps, our characterizations of density order of quasi-projection operators and of a Parseval wavelet frame from the OEP are the main novelty of this paper.

The following result is a characterization of density order provided by the operators $P_{\phi, A, n}$. 
Theorem 2. Let $\phi \in L^{2}\left(\mathbb{R}^{d}\right)$ such that $[\widehat{\phi}, \widehat{\phi}]$ is essentially bounded. The operators $P_{\phi, A, n}, n \in \mathbb{N}$, provide A-density order $\alpha$ if and only if the two following conditions hold:

(i) $\Lambda_{\phi, A, \alpha}$ and $\Gamma_{\phi, A, \alpha}$ are in $L^{\infty}\left(\mathbb{R}^{d}\right)$;

(ii) the origin is a point of $A^{*}$-approximate continuity of both $\Lambda_{\phi, A, \alpha}$ and of $\Gamma_{\phi, A, \alpha}$.

With respect to density order provided by a Parseval wavelet frame, we prove the following consequence.

Corollary 2. With the same hypotheses as in Theorem A. The Parseval wavelet frame $\left\{\psi^{(\ell)}: 1 \leq \ell \leq N\right\}$ provides A-density order $\alpha$ if and only if the two following conditions hold:

(i) $\Lambda_{\sqrt{S} \phi, A, \alpha}$ and $\Gamma_{\sqrt{S} \phi, A, \alpha}$ are in $L^{\infty}\left(\mathbb{R}^{d}\right)$;

(ii) the origin is a point of $A^{*}$-approximate continuity of $\Lambda_{\sqrt{S} \phi, A, \alpha}$ and of $\Gamma \sqrt{S} \phi, A, \alpha$.

The function $\Lambda_{\phi, A, \alpha}$ appears in our results in order to use the same notation in the characterization of approximation order and density order provided by a shift invariant subspace given in [4].

\section{Proofs of the main Results.}

Mainly, ideas for our proofs have been developed in [23], [24] and [14].

4.1. Proof of Theorem 1 and Corollary 1. For the proof of Theorem 1, we need two previous lemmas to estimate the difference between $f$ and $P_{\phi, A, n}(f)$ in $L^{2}\left(\mathbb{R}^{d}\right)$ norm.

We consider the same notation as in the statement of Theorem 1. Define the set $I_{A}:=\bigcup_{l=0}^{\infty} A^{*-l} B_{r_{0}}$ with a fixed $r_{0}>0$ for which $I_{A} \subset(-1 / 2,1 / 2)^{d}$. This $r_{0}$ exists because $A^{*}$ is expansive. Let $f_{n} \in L^{2}\left(\mathbb{R}^{d}\right)$ such that $\widehat{f_{n}}=\widehat{f} \chi_{A^{* n} I_{A}}$. Then

$$
\begin{aligned}
& \left\|f-P_{\phi, A, n}(f)\right\|_{L^{2}\left(\mathbb{R}^{d}\right)} \\
\leq & \left\|f-f_{n}\right\|_{L^{2}\left(\mathbb{R}^{d}\right)}+\left\|f_{n}-P_{\phi, A, n}\left(f_{n}\right)\right\|_{L^{2}\left(\mathbb{R}^{d}\right)}+\left\|P_{\phi, A, n}\left(f_{n}\right)-P_{\phi, A, n}(f)\right\|_{L^{2}\left(\mathbb{R}^{d}\right)} \\
(1) \leq & K\left\|f-f_{n}\right\|_{L^{2}\left(\mathbb{R}^{d}\right)}+\left\|f_{n}-P_{\phi, A, n}\left(f_{n}\right)\right\|_{L^{2}\left(\mathbb{R}^{d}\right)},
\end{aligned}
$$

where $K$ is a non negative constant. The last inequality is true because $P_{\phi, A, n}$ is linear and bounded. Indeed, $K$ may be taken as $K=1+\left\|P_{\phi, A, n}\right\| \leq 1+$ $\|[\widehat{\phi}, \widehat{\phi}]\|_{L^{\infty}\left(\mathbb{T}^{d}\right)}$.

In the two following lemmas, we estimate the sum in the right hand of (1). First, we need a version of [11, Lemma 5.1].

Lemma B. There exists $C>0$ such that for any $f \in W_{A}^{\alpha, 2}\left(\mathbb{R}^{d}\right)$ and $n \in \mathbb{N}$

$$
\left\|\left(1-\chi_{A^{* n} I_{A}}\right) \widehat{f}\right\|_{L^{2}\left(\mathbb{R}^{d}\right)} \leq C \kappa_{n, f} d_{A}^{-n \alpha / d}\|f\|_{W_{A}^{\alpha, 2}\left(\mathbb{R}^{d}\right)},
$$

where $0 \leq \kappa_{n, f} \leq 1$ is constant depending of $n$ and $f$. In addition, $\lim _{n \rightarrow \infty} \kappa_{n, f}=0$.

We also need the lemma below. 
Lemma 1. Let $f \in L^{2}\left(\mathbb{R}^{d}\right)$ and $n \in \mathbb{N}$, then

$$
\begin{aligned}
& \left\|f_{n}-P_{\phi, A, n}\left(f_{n}\right)\right\|_{L^{2}\left(\mathbb{R}^{d}\right)}^{2} \\
& =\left.\left.\int_{A^{* n} I_{A}}|\widehat{f}(\mathbf{t})|^{2}|1-| \widehat{\phi}\left(A^{*-n} \mathbf{t}\right)\right|^{2}\right|^{2} d \mathbf{t} \\
& \quad+\left.\int_{A^{* n} I_{A}}\left|\widehat{f}(\mathbf{t}) \widehat{\phi}\left(A^{*-n} \mathbf{t}\right)\right|^{2}\left|[\widehat{\phi}, \widehat{\phi}]\left(A^{*-n} \mathbf{t}\right)-\right| \widehat{\phi}\left(A^{*-n} \mathbf{t}\right)\right|^{2} \mid d \mathbf{t} .
\end{aligned}
$$

Proof. By Parseval equality,

$$
\begin{aligned}
P_{\phi, A, n}\left(f_{n}\right)(\mathbf{t}) & =\sum_{\mathbf{k} \in \mathbb{Z}^{d}}\left\langle D_{A^{*}}^{n} \widehat{f_{n}} \bar{\phi}, e^{-2 \pi i \mathbf{k} \cdot}\right\rangle e^{-2 \pi i \mathbf{k} \cdot\left(A^{*}\right)^{-n} \mathbf{t}} D_{A^{*}}^{-n} \widehat{\phi}(\mathbf{t}) \\
& =\left[D_{A^{*}}^{n} \widehat{f_{n}}, \widehat{\phi}\right]\left(A^{*-n} \mathbf{t}\right) D_{A^{*}}^{-n} \widehat{\phi}(\mathbf{t}) \\
& =\sum_{\mathbf{k} \in \mathbb{Z}^{d}} d_{A}^{n / 2} \widehat{f_{n}}\left(\mathbf{t}+\left(A^{*}\right)^{n} \mathbf{k}\right) \widehat{\phi}\left(A^{*-n} \mathbf{t}+\mathbf{k}\right) D_{A^{*}}^{-n} \widehat{\phi}(\mathbf{t}),
\end{aligned}
$$

where the second equality holds because $\left[D_{A^{*}}^{n} \widehat{f_{n}}, \widehat{\phi}\right] \in L^{2}\left(\mathbb{T}^{d}\right)$. Thus, we have

$$
\begin{aligned}
& \left\|f_{n}-P_{\phi, A, n}\left(f_{n}\right)\right\|_{L^{2}\left(\mathbb{R}^{d}\right)}^{2}=\left\|\widehat{f_{n}}-\widehat{P_{\phi, A, n}\left(f_{n}\right)}\right\|_{L^{2}\left(\mathbb{R}^{d}\right)}^{2} \\
= & \int_{\mathbb{R}^{d}}\left|\widehat{f_{n}}(\mathbf{t})-\left[D_{A^{*}}^{n} \widehat{f_{n}}, \widehat{\phi}\right]\left(A^{*-n} \mathbf{t}\right) D_{A^{*}}^{-n} \widehat{\phi}(\mathbf{t})\right|^{2} d \mathbf{t} \\
= & \int_{\mathbb{R}^{d}}\left|\widehat{f_{n}}(\mathbf{t})\right|^{2} d \mathbf{t}-\int_{\mathbb{R}^{d}} \widehat{f_{n}}(\mathbf{t}) \overline{\left[D_{A^{*}}^{n} \widehat{f_{n}}, \widehat{\phi}\right]\left(A^{*-n} \mathbf{t}\right) D_{A^{*}}^{-n} \widehat{\phi}(\mathbf{t})} d \mathbf{t} \\
& -\int_{\mathbb{R}^{d}} \widehat{\widehat{f_{n}}(\mathbf{t})}\left[D_{A^{*}}^{n} \widehat{f_{n}}, \widehat{\phi}\right]\left(A^{*-n} \mathbf{t}\right) D_{A^{*}}^{-n} \widehat{\phi}(\mathbf{t}) d \mathbf{t} \\
& +\int_{\mathbb{R}^{d}}\left|\left[D_{A^{*}}^{n} \widehat{f_{n}}, \widehat{\phi}\right]\left(A^{*-n} \mathbf{t}\right) D_{A^{*}}^{-n} \widehat{\phi}(\mathbf{t})\right|^{2} d \mathbf{t} .
\end{aligned}
$$

Since $\left[D_{A^{*}}^{n} \widehat{f_{n}}, \widehat{\phi}\right]\left(A^{*-n} \mathbf{t}\right)$ is $\left(A^{*}\right)^{n} \mathbb{Z}^{d}$-periodic, we can write

$$
\begin{aligned}
& \int_{\mathbb{R}^{d}} \widehat{f_{n}}(\mathbf{t}) \overline{\left[D_{A^{*}}^{n} \widehat{f_{n}}, \widehat{\phi}\right]\left(A^{*-n} \mathbf{t}\right) D_{A^{*}}^{-n} \widehat{\phi}(\mathbf{t})} d \mathbf{t} \\
& =\sum_{\mathbf{m} \in \mathbb{Z}^{\mathbf{d}}} \int_{\left(A^{*}\right)^{n}\left[-\frac{1}{2}, \frac{1}{2}\right]^{d}+\left(A^{*}\right)^{n} \mathbf{m}} \widehat{f_{n}}(\mathbf{t}) \overline{\left[D_{A^{*}}^{n} \widehat{f_{n}}, \widehat{\phi}\right]\left(A^{*-n} \mathbf{t}\right) D_{A^{*}}^{-n} \widehat{\phi}(\mathbf{t})} d \mathbf{t} \\
& =d_{A}^{-n} \int_{\left(A^{*}\right)^{n}\left[-\frac{1}{2}, \frac{1}{2}\right]^{d}}\left|\left[D_{A^{*}}^{n} \widehat{f_{n}}, \widehat{\phi}\right]\left(A^{*-n} \mathbf{t}\right)\right|^{2} d \mathbf{t} \\
& =\int_{\left(A^{*}\right)^{n} I_{A}}\left|\widehat{f}(\mathbf{t}) \widehat{\phi}\left(A^{*-n} \mathbf{t}\right)\right|^{2} d \mathbf{t},
\end{aligned}
$$

where the last equality is true because $\widehat{f_{n}}=\widehat{f} \chi_{\left(A^{*}\right)^{n} I_{A}}$ and $I_{A} \subset\left[-\frac{1}{2}, \frac{1}{2}\right]^{d}$.

In an analogous way, we obtain

$$
\begin{aligned}
& \int_{\mathbb{R}^{d}}\left|\left[D_{A^{*}}^{n} \widehat{f_{n}}, \widehat{\phi}\right]\left(A^{*-n} \mathbf{t}\right)\right|^{2} \mid D_{A^{*}}^{-n} \widehat{\left.\phi(\mathbf{t})\right|^{2}} d \mathbf{t} \\
= & \int_{\left(A^{*}\right)^{n} I_{A}}\left|\widehat{f}(\mathbf{t}) \widehat{\phi}\left(A^{*-n} \mathbf{t}\right)\right|^{2}[\widehat{\phi}, \widehat{\phi}]\left(A^{*-n} \mathbf{t}\right) d \mathbf{t}
\end{aligned}
$$


By (2), (3) and (4), we conclude that

$$
\begin{aligned}
& \left\|f_{n}-P_{\phi, A, n}\left(f_{n}\right)\right\|_{L^{2}\left(\mathbb{R}^{d}\right)}^{2} \\
= & \int_{\left(A^{*}\right)^{n} I_{A}}|\widehat{f}(\mathbf{t})|^{2} d \mathbf{t}-2 \int_{\left(A^{*}\right)^{n} I_{A}}\left|\widehat{f}(\mathbf{t}) \widehat{\phi}\left(A^{*-n} \mathbf{t}\right)\right|^{2} d \mathbf{t} \\
& +\int_{\left(A^{*}\right)^{n} I_{A}}\left|\widehat{f}(\mathbf{t}) \widehat{\phi}\left(A^{*-n} \mathbf{t}\right)\right|^{2}[\widehat{\phi}, \widehat{\phi}]\left(A^{*-n} \mathbf{t}\right) d \mathbf{t} \\
= & \left.\left.\int_{\left(A^{*}\right)^{n} I_{A}}|\widehat{f}(\mathbf{t})|^{2}|1-| \widehat{\phi}\left(A^{*-n} \mathbf{t}\right)\right|^{2}\right|^{2} d \mathbf{t} \\
& +\left.\int_{\left(A^{*}\right)^{n} I_{A}}\left|\widehat{f}(\mathbf{t}) \widehat{\phi}\left(A^{*-n} \mathbf{t}\right)\right|^{2}\left|[\widehat{\phi}, \widehat{\phi}]\left(A^{*-n} \mathbf{t}\right)-\right| \widehat{\phi}\left(A^{*-n} \mathbf{t}\right)\right|^{2} \mid d \mathbf{t} .
\end{aligned}
$$

Proof of Theorem 1. First, observe that when $\alpha=0$ the result is trivial. Assume that $\alpha>0$. Let us prove the sufficient condition. Bearing in mind that $[\widehat{\phi}, \widehat{\phi}] \in$ $L^{\infty}\left(\mathbb{T}^{d}\right)$, the functions $\Gamma_{\phi, A, \alpha}, \Lambda_{\phi, A, \alpha} \in L^{\infty}\left(\mathbb{R}^{d}\right)$ and Lemma 1 , we obtain

$$
\begin{aligned}
& \quad\left\|f_{n}-P_{\phi, A, n}\left(f_{n}\right)\right\|_{L^{2}\left(\mathbb{R}^{d}\right)}^{2}=\int_{A^{* n} I_{A}}|\widehat{h}(\mathbf{t})|^{2} \frac{\left.\left.|1-| \widehat{\phi}\left(A^{*-n} \mathbf{t}\right)\right|^{2}\right|^{2}}{\left(1+d_{A}^{n / d} \rho\left(A^{*-n} \mathbf{t}\right)\right)^{2 \alpha}} d \mathbf{t} \\
& \quad+\int_{\left(A^{*}\right)^{n} I_{A}}|\widehat{h}(\mathbf{t})|^{2}\left|\widehat{\phi}\left(A^{*-n} \mathbf{t}\right)\right|^{2} \frac{\left.\left|[\widehat{\phi}, \widehat{\phi}]\left(A^{*-n} \mathbf{t}\right)-\right| \widehat{\phi}\left(A^{*-n} \mathbf{t}\right)\right|^{2} \mid}{\left(1+d_{A}^{n / d} \rho\left(A^{*-n} \mathbf{t}\right)\right)^{2 \alpha}} d \mathbf{t} \\
& \leq d_{A}^{-2 n \alpha / d}\left(\left\|\Gamma_{\phi, A, \alpha}\right\|_{L^{\infty}\left(\mathbb{R}^{d}\right)}^{2 \alpha}+\|[\widehat{\phi}, \widehat{\phi}]\|_{L^{\infty}\left(\mathbb{T}^{d}\right)}^{2}\left\|\Lambda_{\phi, A, \alpha}\right\|_{L^{\infty}\left(\mathbb{R}^{d}\right)}^{2}\right)\|f\|_{W_{A}^{\alpha, 2}\left(\mathbb{R}^{d}\right)}^{2},
\end{aligned}
$$

where $\widehat{h}=(1+\rho)^{\alpha} \widehat{f}$. Therefore, according to (1) and Lemma B, we get that $P_{\phi, A, n}$ provides $A$-approximation order $\alpha$.

Now, let us prove the necessity condition. By hypothesis, there exists $C>0$ such that for any $f \in W_{A}^{\alpha, 2}\left(\mathbb{R}^{d}\right)$ and $n \in \mathbb{N}$ we have

$$
\left\|f_{n}-P_{\phi, A, n}\left(f_{n}\right)\right\|_{L^{2}\left(\mathbb{R}^{d}\right)}^{2} \leq C^{2} d_{A}^{-2 n \alpha / d}\left\|f_{n}\right\|_{W_{A}^{\alpha, 2}\left(\mathbb{R}^{d}\right)}^{2} .
$$

Observe that when $f$ ranges over all functions in $W_{A}^{\alpha, 2}\left(\mathbb{R}^{d}\right),|\widehat{h}|^{2}=\left|(1+\rho)^{\alpha} \widehat{f}\right|^{2}$ runs over all non negative functions in $L^{1}\left(\mathbb{R}^{d}\right)$. According to Lemma 1 , the last inequality implies that

$$
\begin{aligned}
& \int_{A^{* n} I_{A}}|\widehat{h}(\mathbf{t})|^{2} \frac{\left.\left.|1-| \widehat{\phi}\left(A^{*-n} \mathbf{t}\right)\right|^{2}\right|^{2}}{(1+\rho(\mathbf{t}))^{2 \alpha}} d \mathbf{t} \\
\leq & \left\|f_{n}-P_{\phi, A, n}\left(f_{n}\right)\right\|_{L^{2}\left(\mathbb{R}^{d}\right)}^{2} \leq C^{2} d_{A}^{-2 n \alpha / d}\left\|f_{n}\right\|_{W_{A}^{\alpha, 2}\left(\mathbb{R}^{d}\right)}^{2} \\
\leq & C^{2} d_{A}^{-2 n \alpha / d}\left\||\widehat{h}|^{2}\right\|_{L^{1}\left(\mathbb{R}^{d}\right)} .
\end{aligned}
$$

Thus

$$
d_{A}^{2 n \alpha / d} \chi_{A^{* n} I_{A}}(\mathbf{t}) \frac{\left.\left.|1-| \widehat{\phi}\left(A^{*-n} \mathbf{t}\right)\right|^{2}\right|^{2}}{(1+\rho(\mathbf{t}))^{2 \alpha}}
$$


may be considered as a continuous linear functional on $L^{1}\left(\mathbb{R}^{d}\right)$ with norm bounded by $C^{2}$. Then

$$
\begin{aligned}
C & \geq\left\|d_{A}^{n \alpha / d} \chi_{A^{* n} I_{A}}(\cdot) \frac{\left.|1-| \widehat{\phi}\left(A^{*-n} \cdot\right)\right|^{2} \mid}{\left(1+d_{A}^{n / d} \rho\left(A^{*-n} \cdot\right)\right)^{\alpha}}\right\|_{L^{\infty}\left(\mathbb{R}^{d}\right)} \\
& =\left\|\chi_{A^{* n} I_{A}}(\cdot) \frac{\left.|1-| \widehat{\phi}\left(A^{*-n} \cdot\right)\right|^{2} \mid}{\left(d_{A}^{-n / d}+\rho\left(A^{*-n} \cdot\right)\right)^{\alpha}}\right\|_{L^{\infty}\left(\mathbb{R}^{d}\right)}
\end{aligned}
$$

Rescaling and having in mind that $d_{A}>1$ we get that $\Gamma_{\phi, A, \alpha}$ is in $L^{\infty}\left(\mathbb{R}^{d}\right)$ by taking $n \rightarrow \infty$.

It remains to check that $\Lambda_{\phi, A, \alpha}$ is in $L^{\infty}\left(\mathbb{R}^{d}\right)$. Since $\Gamma_{\phi, A, \alpha}$ is in $L^{\infty}\left(\mathbb{R}^{d}\right)$, we have that there exists $U \subset \mathbb{R}^{d}$, a neighborhood of the origin such that $|\widehat{\phi}(\mathbf{t})| \geq \frac{1}{2}$ for $\mathbf{t} \in U$. Now we proceed similarly as above to get that

$$
C \geq\left\||\widehat{\phi}(\cdot)|^{2} \frac{\left.|[\widehat{\phi}, \widehat{\phi}](\cdot)-| \widehat{\phi}(\cdot)\right|^{2} \mid}{\rho(\cdot)^{\alpha}}\right\|_{L^{\infty}(U)} \geq \frac{1}{2^{4}}\left\|\frac{\left|1-\frac{|\widehat{\phi}(\cdot)|^{2}}{[\widehat{\phi}, \widehat{\phi}](\cdot)}\right|}{\rho(\cdot)^{\alpha}}\right\|_{L^{\infty}(U)},
$$

for some $C>0$. Hence the proof is finished.

To prove Corollary 1, we will use the following result which is implicit in different proofs of the Unitary Extension Principle. When the dilation matrix is the dyadic, see e.g. [14, Lemma 2.4],[9, pag. 322], [15, pag. 36]. For more general dilation, see $[14$, Lemma 5.5] and [1].

Lemma C. With the same notation as in Theorem A. Then

$$
P_{\sqrt{S} \phi, A, n}(f)=Q_{\phi, A, n}(f), \quad \forall f \in W_{A}^{2, \alpha}\left(\mathbb{R}^{d}\right),
$$

where the equality holds in $L^{2}\left(\mathbb{R}^{d}\right)$ sense.

Proof of Corollary 1. Let $f \in W_{A}^{\alpha, 2}\left(\mathbb{R}^{d}\right)$ and $n_{0} \in \mathbb{N}$. According to Lemma C, we have

$$
\left\|f-Q_{\Psi, A, n_{0}}(f)\right\|_{L^{2}\left(\mathbb{R}^{d}\right)}=\left\|f-P_{\sqrt{S} \phi, A, n_{0}}(f)\right\|_{L^{2}\left(\mathbb{R}^{d}\right)} .
$$

It means that $\Psi$ provides $A$-approximation order $\alpha$ is equivalent to the operators $P_{\sqrt{S} \phi, A, n}, n \in \mathbb{N}$, provide $A$-approximation order $\alpha$. Hence, by Theorem 1 we get the statement of Corollary 1.

4.2. Proof of Theorem 2 and Corollary 2. For the proof of Theorem 2 we need the following proposition that is a version of [11, Proposition 5.3].

Proposition D. Let $f: \mathbb{R}^{d} \rightarrow \mathbb{R}$ be a measurable function such that $f(\mathbf{0})=0$ and $0 \leq f(\mathbf{x}) \leq M$ for a.e. $\mathbf{x} \in \mathbb{R}^{d}$ and some $M>0$. The following conditions are equivalent:

i) The origin is a point of $A^{*}$-approximate continuity of $f$.

ii) There exists $K \subset \mathbb{R}^{d}$ a bounded and measurable set containing a neighborhood of the origin such that

$$
\lim _{j \rightarrow \infty} \frac{1}{\left|A^{*-j} K\right|} \int_{A^{*-j} K} f(\mathbf{x}) d \mathbf{x}=0 .
$$

iii) For all $K \subset \mathbb{R}^{d}$ bounded and measurable (5) holds.

We are ready to prove the second theorem of this paper. 
Proof of Theorem 2. Let us prove the sufficiency. For any given $n \in \mathbb{N}$, we set

$$
\Pi_{n}(\mathbf{t})=d_{A}^{2 n \alpha / d} \chi_{A^{* n} I_{A}}(\mathbf{t}) \frac{\left.\left.|1-| \widehat{\phi}\left(A^{*-n} \mathbf{t}\right)\right|^{2}\right|^{2}}{(1+\rho(\mathbf{t}))^{2 \alpha}}, \quad \mathbf{t} \in \mathbb{R}^{d} .
$$

Since $\Gamma_{\phi, A, \alpha} \in L^{\infty}\left(\mathbb{R}^{d}\right)$, we have $0 \leq \Pi_{n}(\mathbf{t}) \leq\left\|\Gamma_{\phi, A, \alpha}^{2}\right\|_{L^{\infty}\left(\mathbb{R}^{d}\right)}$ a.e. So, $\Pi_{n}$ may be considered as an element of the dual space of $L^{1}\left(\mathbb{R}^{d}\right)$.

Let $K \subset \mathbb{R}^{d}$ be a compact set. Since $A^{*}$ is expansive, there exists $n_{0} \in \mathbb{N}$ such that if $n \geq n_{0}$, then $A^{*-n} K \subset I_{A}$. Therefore, for those $n$, we have

$$
\begin{aligned}
\Pi_{n}\left(\chi_{K}\right) & =d_{A}^{2 n \alpha / d} \int_{K} \frac{\left.\left.|1-| \widehat{\phi}\left(A^{*-n} \mathbf{t}\right)\right|^{2}\right|^{2}}{(1+\rho(\mathbf{t}))^{2 \alpha}} d \mathbf{t} \\
& =d_{A}^{2 n \alpha / d} d_{A}^{n} \int_{A^{*-n} K} \frac{\left.\left.|1-| \widehat{\phi}(\mathbf{s})\right|^{2}\right|^{2}}{\left(1+\rho\left(A^{* n} \mathbf{s}\right)\right)^{2 \alpha}} d \mathbf{s} \\
& =d_{A}^{n} \int_{A^{*-n} K} \frac{\left.\left.|1-| \widehat{\phi}(\mathbf{s})\right|^{2}\right|^{2}}{\left(d_{A}^{-n / d}+\rho(\mathbf{s})\right)^{2 \alpha}} d \mathbf{s} .
\end{aligned}
$$

By hypotheses we know that origin is a point of $A^{*}$-approximate continuity of $\Gamma_{\phi, A, \alpha}$, then Proposition D and (6) deal to

$$
0 \leq \lim _{n \rightarrow \infty} \Pi_{n}\left(\chi_{K}\right) \leq \lim _{n \rightarrow \infty} \frac{|K|}{\left|A^{*-n} K\right|} \int_{A^{*-n} K} \Gamma_{\phi, A, \alpha}^{2}(\mathbf{t}) d \mathbf{t}=0 .
$$

Thus, by linearity, $\lim _{j \rightarrow \infty} \Pi_{j}(u)=0$ for any simple function $u$. Furthermore by a density argument,

$$
\lim _{n \rightarrow \infty} \Pi_{n}(g)=0, \quad \forall g \in L^{1}\left(\mathbb{R}^{d}\right) .
$$

On other hand, we consider

$$
\Upsilon_{n}(\mathbf{t})=d_{A}^{2 n \alpha / d} \chi_{A^{* n} I_{A}}(\mathbf{t})\left|\widehat{\phi}\left(A^{*-n} \mathbf{t}\right)\right|^{2} \frac{\mid \widehat{\phi}, \widehat{\phi}]\left(A^{*-n} \mathbf{t}\right)-\left|\widehat{\phi}\left(A^{*-n} \mathbf{t}\right)\right|^{2} \mid}{(1+\rho(\mathbf{t}))^{2 \alpha}}, \quad \mathbf{t} \in \mathbb{R}^{d} .
$$

Since $\Lambda_{\phi, A, \alpha} \in L^{\infty}\left(\mathbb{R}^{d}\right)$, we have $0 \leq \Upsilon_{n}(\mathbf{t}) \leq\|[\widehat{\phi}, \widehat{\phi}]\|_{L^{\infty}\left(\mathbb{T}^{d}\right)}^{2}\left\|\Lambda_{\phi, A, \alpha}^{2}\right\|_{L^{\infty}\left(\mathbb{R}^{d}\right)}$ a.e. So, $\Upsilon_{n}$ may be considered as an element of the dual space of $L^{1}\left(\mathbb{R}^{d}\right)$.

Following the same argument to obtain (7), we get

$$
\lim _{n \rightarrow \infty} \Upsilon_{n}(g)=0, \quad \forall g \in L^{1}\left(\mathbb{R}^{d}\right) .
$$

By Lemma 1 and (8),

$$
\begin{aligned}
& \lim _{n \rightarrow \infty} d_{A}^{2 n \alpha / d}\left\|f_{n}-P_{\phi, A, n}\left(f_{n}\right)\right\|_{L^{2}\left(\mathbb{R}^{d}\right)}^{2} \\
= & \lim _{n \rightarrow \infty}\left(\Pi_{n}\left((1+\rho)^{2 \alpha}|\widehat{f}|^{2}\right)+\Upsilon_{n}\left((1+\rho)^{2 \alpha}|\widehat{f}|^{2}\right)\right)=0, \quad \forall f \in W_{A}^{\alpha, 2}\left(\mathbb{R}^{d}\right) .
\end{aligned}
$$

This together (1) and Lemma B conclude that the linear operators $P_{\phi, A, n}$ provide $A$-density order $\alpha$, as we wanted to see.

Now we prove the necessity condition. We assume that the linear operators $P_{\phi, A, n}$ provide $A$-density order $\alpha$. By Remark 2 , they also provide $A$-approximation order $\alpha$. Thus, according to Theorem 1 , we have that $\Gamma_{\phi, A, \alpha}$ and $\Lambda_{\phi, A, \alpha}$ are in $L^{\infty}\left(\mathbb{R}^{d}\right)$. 
Let us fix a particular $f \in W_{A}^{\alpha, 2}\left(\mathbb{R}^{d}\right)$ defined by $\widehat{f}(\mathbf{t})=\chi_{I_{A}}(\mathbf{t})(1+\rho(\mathbf{t}))^{-\alpha}$. Using that $I_{A} \subset A^{* n} I_{A}, n \in \mathbb{N}$, and Lemma 1 , we obtain

$$
0=\lim _{n \rightarrow \infty} \frac{d_{A}^{2 n \alpha / d}}{\left|I_{A}\right|}\left\|f-P_{\phi, A, n}(f)\right\|_{L^{2}\left(\mathbb{R}^{d}\right)}^{2}=\lim _{n \rightarrow \infty} M(n),
$$

where

$$
\begin{aligned}
M(n)= & \frac{1}{\left|A^{*-n} I_{A}\right|} \int_{A^{*-n} I_{A}} \frac{\left.\left.|1-| \widehat{\phi}(\mathbf{t})\right|^{2}\right|^{2}}{\left(d_{A}^{-n / d}+\rho(\mathbf{t})\right)^{2 \alpha}} \\
& +|\widehat{\phi}(\mathbf{t})|^{2} \frac{\left.|[\widehat{\phi}, \widehat{\phi}](\mathbf{t})-| \widehat{\phi}(\mathbf{t})\right|^{2} \mid}{\left(d_{A}^{-n / d}+\rho(\mathbf{t})\right)^{2 \alpha}} d \mathbf{t} .
\end{aligned}
$$

On other hand, let $n, \ell \in \mathbb{N}$. Since there exists a constant $C>0$ such that $\rho(\mathbf{t})^{-2 \alpha} \leq C(1+\rho(\mathbf{t}))^{-2 \alpha}, \forall \mathbf{t} \in I_{A} \backslash A^{*-1} I_{A}$, we obtain the following estimation

$$
\begin{aligned}
& \frac{1}{\left|A^{*-n-\ell} I_{A}\right|} \int_{A^{*-(n+\ell)}\left(I_{A} \backslash A^{*-1} I_{A}\right)} \Gamma_{\phi, A, \alpha}^{2}(\mathbf{t}) d \mathbf{t} \\
= & \frac{1}{\left|I_{A}\right|} \int_{I_{A} \backslash A^{*-1} I_{A}} \frac{\left.\left.|1-| \widehat{\phi}\left(A^{*-(n+\ell)} \mathbf{s}\right)\right|^{2}\right|^{2}}{\left(d_{A}^{-(n+\ell) / d} \rho(\mathbf{s})\right)^{2 \alpha}} d \mathbf{s} \\
\leq & \frac{C}{\left|A^{*-n-\ell} I_{A}\right|} \int_{A^{*-(n+\ell)}\left(I_{A} \backslash A^{*-1} I_{A}\right)} \frac{\left.\left.|1-| \widehat{\phi}(\mathbf{t})\right|^{2}\right|^{2}}{\left(d_{A}^{-(n+\ell) / d}+\rho(\mathbf{t})\right)^{2 \alpha}} d \mathbf{t} .
\end{aligned}
$$

By (10) and (9), we get

$$
\begin{aligned}
0 & \leq \lim _{n \rightarrow \infty} \frac{1}{\left|A^{*-n} I_{A}\right|} \int_{A^{*-n} I_{A}} \Gamma_{\phi, A, \alpha}^{2}(\mathbf{t}) d \mathbf{t} \\
& =\lim _{n \rightarrow \infty} \sum_{\ell=0}^{\infty} \frac{d_{A}^{-\ell}}{\left|A^{*-n-\ell} I_{A}\right|} \int_{A^{*-(n+\ell)}\left(I_{A} \backslash A^{*-1} I_{A}\right)} \Gamma_{\phi, A, \alpha}^{2}(\mathbf{t}) d \mathbf{t} \\
& \leq \lim _{n \rightarrow \infty} \sum_{\ell=0}^{\infty} \frac{C d_{A}^{-\ell}}{\left|A^{*-n-\ell} I_{A}\right|} \int_{A^{*-(n+\ell)}\left(I_{A} \backslash A^{*-1} I_{A}\right)} \frac{\left.\left.|1-| \widehat{\phi}(\mathbf{t})\right|^{2}\right|^{2}}{\left(d_{A}^{-(n+\ell) / d}+\rho(\mathbf{t})\right)^{2 \alpha}} d \mathbf{t} \\
& \leq \lim _{n \rightarrow \infty} C \sum_{\ell=0}^{\infty} M(n+\ell) d_{A}^{-\ell}=0 .
\end{aligned}
$$

By Proposition D, we conclude that the origin is a point of $A^{*}$-approximate continuity of $\Gamma_{\phi, A, \alpha}$.

Similarly, we have

$$
\lim _{n \rightarrow \infty} \frac{1}{\left|A^{*-n} I_{A}\right|} \int_{A^{*-n} I_{A}}|\widehat{\phi}(\mathbf{t})|^{2}[\widehat{\phi}, \widehat{\phi}](\mathbf{t}) \Lambda_{\phi, A, \alpha}^{2}(\mathbf{t}) d \mathbf{t}=0 .
$$

Again by Proposition $\mathrm{D}$, we have that the origin is a point of $A^{*}$-approximate continuity of $|\widehat{\phi}(\mathbf{t})|^{2}[\widehat{\phi}, \widehat{\phi}](\mathbf{t}) \Lambda_{\phi, A, \alpha}^{2}(\mathbf{t})$, provided $|\widehat{\phi}(\mathbf{0})|^{2}=1$ and $[\widehat{\phi}, \widehat{\phi}](\mathbf{0})=1$. Now, since the origin is a point of $A^{*}$-approximate continuity of $\Gamma_{\phi, A, \alpha}$, then the origin is a point of $A^{*}$-approximate continuity of $|\widehat{\phi}(\mathbf{t})|^{2}$. Hence the origin is a point of $A^{*}$-approximate continuity of $\Lambda_{\phi, A, \alpha}$. This finishes the proof. 
We are ready to prove Corollary 2 .

Proof of Corollary 2. Let $f \in W_{A}^{\alpha, 2}\left(\mathbb{R}^{d}\right)$ and $n_{0} \in \mathbb{N}$. According to Lemma C, we have

$$
\left\|f-Q_{\Psi, A, n_{0}}(f)\right\|_{L^{2}\left(\mathbb{R}^{d}\right)}=\left\|f-P_{\sqrt{S} \phi, A, n_{0}}(f)\right\|_{L^{2}\left(\mathbb{R}^{d}\right)} .
$$

It means that $\Psi$ provides $A$-density order $\alpha$ is equivalent to the operators $P_{\sqrt{S} \phi, A, n}$, $n \in \mathbb{N}$, provide $A$-density order $\alpha$. Thus, by Theorem 1 , the proof is finished.

\section{REFERENCES}

[1] N. Atreas, A. Melas, T. Stavropoulos; Affine dual frames and extension principles, Appl. Comput. Harmon. Anal. 36 (2014), No. 1, 51-62.

[2] J.J. Benedetto, S. Li; The theory of multiresolution analysis frames and applications to filter banks, Appl. Comput. Harmon. Anal. 5 (1998), no. 4, 389-427.

[3] C. de Boor, R.A. DeVore, A. Ron; Approximation orders of FSI spaces in $L^{2}\left(\mathbb{R}^{d}\right)$, Constr. Approx. 14 (1998), no. 4, 631-652.

[4] C. de Boor, R.A. DeVore, A. Ron; Approximation from shift-invariant subspaces of $L^{2}\left(\mathbb{R}^{d}\right)$, Trans. Amer. Math. Soc. 341 (1994), no. 2, 787-806.

[5] M. Bownik, Z. Rzeszotni; The spectral function of shift-invariant spaces, Michigan Math. J. 51 (2003), no. 2, 387-414.

[6] A. Bruckner; Differentiation of real functions, Lecture Notes in Mathematics, 659. Springer, Berlin, 1978.

[7] C.K. Chui, W. He, J. Stöckler Compactly supported tight and sibling frames with maximum vanishing moments, Appl. Comput. Harmonic Anal. 13 (2002) 224-262.

[8] D. R. Chen, X. Zheng; Stability implies convergence of cascade algorithms in Sobolev space, J. Math. Anal. Appl. 268 (2002), no. 1, 41-52..

[9] O. Christensen; An Introduction to frames and Riesz bases, Birkhäser, Boston, 2003.

[10] P. Cifuentes, K. S. Kazarian, A. San Antolín; Characterization of scaling functions in a multiresolution analysis, Proc. Amer. Math. Soc. 133 (2005), no. 4, 1013-1023.

[11] P. Cifuentes, A. San Antolin, M. Soto-Bajo; Anisotropic dilations of shift-invariant subspaces and approximation properties in $L^{2}\left(\mathbb{R}^{d}\right)$, Math. Nachr. 288, (2015) No. 5-6, 525-539.

[12] A. Cohen, K. Grochenig, L.F. Villemoes; Regularity of multivariate refinable functions, Constructive Approximation 15 (1999), No. 2, 241-255.

[13] I. Daubechies; Ten lectures on wavelets, SIAM, Philadelphia, 1992.

[14] I. Daubechies, B. Han, A. Ron, Z.Shen; Framelets: MRA-based constructions of wavelet frames, Appl. Comput. Harmon. Anal. 14 (2003), no. 1, 1-46.

[15] B. Dong, Z. Shen; MRA-based wavelet frames and applications, Mathematics in image processing, American Mathematical Society, Providence, RI, 2013.

[16] H.G. Feichtinger, N. Kaiblinger; Quasi-interpolation in the Fourier algebra, J. Approx. Theory 144 (2007), No. 1, 103-118.

[17] K. Gröchenig, W.R. Madych; Multiresolution analysis, Haar bases, and self-similar tilings of $\mathbb{R}^{n}$, IEEE Trans. Inf. Theory 38 (1992), No. 2, Part II, 556-568.

[18] B. Han; Framelets and wavelets. Algorithms, analysis, and applications, Applied and Numerical Harmonic Analysis. Cham: Birkhuser xxxiii, 724 p. (2017).

[19] B. Han Nonhomogeneous wavelet systems in high dimensions, App. Comput. Harmon. Anal. 32 (2012), no. 2, 169-196.

[20] B. Han; Pairs of frequency-based nonhomogeneous dual wavelet frames in the distribution space, Appl. Comput. Harmon. Anal. 29 (2010), no. 3, 330-353.

[21] B. Han, Approximation properties and construction of Hermite interpolants and biorthogonal multiwavelets, J. Approx. Theory 110 (2001), no. 1, 18-53.

[22] B. Han, Z. Shen, Compactly supported symmetric $C^{\infty}$ wavelets with spectral approximation order, SIAM J. Math. Anal. 40 (2008), no. 3, 905-938.

[23] K. Jetter; Multivariate approximation from the cardinal interpolation point of view, Approximation theory VII (Austin, TX, 1992), 131-161, Academic Press, Boston, MA, 1993.

[24] K. Jetter, D.X. Zhou; Order of linear approximation from shift-invariant spaces, Constr. Approx. 11 (1995), no. 4, 423-438. 
[25] R.Q. Jia; Approximation by quasi-projection operators in Besov spaces, J. approx. theory, $162(2010), 1,186-200$.

[26] R.Q. Jia; Approximation with scaled shift-invariant spaces by means of quasi-projection operators, J. Approx. Theory 131 (2004), no. 1, 30-46.

[27] R.Q. Jia; Approximation properties of multivariate wavelets, Math. Comp. 67 (1998), no. $222,647-665$.

[28] R.Q. Jia, C.A. Micchelli; Using the refinement equations for the construction of pre-wavelets. II. Powers of two, Curves and surfaces (Chamonix-Mont-Blanc, 1990), 209-246, Academic Press, Boston, MA, 1991.

[29] Yu. Kolomoitsev, M.Skopina; Quasi-projection operators in weighted $L_{p}$ spaces, Appl. Comput. Harmon. Anal. (2020) https://doi.org/10.1016/j.acha.2020.01.003.

[30] A. Krivoshein, V. Protasov, M. Skopina; Multivariate wavelet frames, Industrial and Applied Mathematics. Singapore: Springer xiii, 248 p. (2016).

[31] A. Krivoshein, M. Skopina; Approximation by frame-like wavelet systems, Appl. Comput. Harmon. Anal. 31 (2011), No. 3, 410-428.

[32] Y-Z Li, J-P Zhang; Extension principles for affine dual frames in reducing subspaces, Appl. Comput. Harmon. Anal. 46, (2019) No. 1, 177-191.

[33] I. P. Natanson; Theory of functions of a real variable, London, vol. II, 1960.

[34] A. Ron, Z. Shen; Affine systems in $L_{2}\left(\mathbb{R}^{d}\right):$ The analysis of the analysis operator, J. Funct. Anal., 148 (1997), 408-447.

[35] A. San Antolín; On Parseval wavelet frames via multiresolution analyses in $H_{G}^{2}$, Can. Math. Bull. 63 (2020), No. 1, 157-172.

[36] A. San Antolín; On density order of the principal shift-invariant subspaces of $L^{2}\left(\mathbb{R}^{d}\right)$, J. Approx. Theory 164 (2012), no. 8, 1007-1025.

Departamento de Matemáticas, Universidad de Alicante, 03080 Alicante, Spain.

E-mail address: angel.sanantolin@ua.es 